to be so precise when fatigue or headache has set the ciliary muscle in a worry.

It is in the public clinic, where much of the detail work must be delegated by the chief, who must yet shoulder the responsibility of it all, that this method of retinoscopy has its most striking value. In one of my services, where a dozen or more refraction measurements had to be made each day, it was easy to give by the ophthalmoscope a rough estimate of the refraction to an assistant, who might be a mere beginner; and when he had finished his subjective tests and marshalled in to the dark-room his patients wearing the correcting lenses in accord with his findings, it took a very few seconds to verify or revise his work in each case and to prescribe the glasses with full assurance of the precise error of refraction. Any error in the position of a cylinder or the strength of it or of a spherical could be at once detected, rectification made, and the result verified. Some men do not desire this accuracy in their hospital work; others will not take even this small trouble to obtain it; but to the conscientious ophthalmologist it is clearly needful, and it will be readily possible by this method of retincscopy.

\section{THE STATUS OF SKIASCOPY.}

Read in the Section on Ophthalmology, at the Forty-fifth Aunual Meeting of the American Medical Association, beld at San Francisco, June $5-8,1894$.

BY H. V. WÜRDEMANN, M.D. MILWAUKEE. WIS.

DIRECTOR WISCONSIN GENERAI HOSPITAI; OCUIIST AND AURIST TO THE CHILDREN'S HOSPITAL, AND TO THE MILWAUKEE HOSPITAL FO DENO INSANE; INSTRECOR IN WYE, EAR AND TMROAT, ELYS HOSPITAL; AND TO THE MILWAUKEE COUNTY TRAINING SCHOOLS, ETC.

It is conceded beyond all doubt by those practiced in the objective methods for determining the ocular refraction, that skiascopy holds the first place as regards accuracy and minuteness of measurement. This you have learned from the special committee appointed last year and empowered to report at this meeting. The sum of our report may be put in one sentence: The objective tests are cssential to the proper examination of the ocular refraction and, although but preliminary to the subjective method, reduce the latter to simple proing of lenses found by the former, eliminating in a great measure dependence on the faltering judgment of the untrained patient, substituting therefor the skill of the expert who, reasoning from scientific data, is thus able to fit glasses in less time and more accurately with consequent satisfaction to himself and his patient. Keratometry, ophthalmoscopy and skiascopy; "the greatest of these is" skiascopy. Thus in a few words you have the standing of the method designated by those familiar with the same.

This paper is not written for these, but for those on the outside who, either through ultra-conservatism, laziness or inability to master its minutiæ, have not yet taken up the shadow test. It is a painful fact that there are men, in other respects good ophthalmologists, whose refraction work is but on a par with that of the glass-selling jeweler and the average optician. They pin their faith to the trial set and to the patient, whose hesitating replies are a constant source of error. As refraction examination forms fully one-half of the oculist's work, such must give way to those skilled in measuring the eye.

The estimation in which skiascopy is held by our eastern confreres is shown by the fact that a whole week is given up to the teaching and application of this method alone at the Philadelphia Polyclinic, in a series of twenty-seven lectures and clinics by a large corps of professors, instructors and clinical assistants.

From the perusal of recent English and German literature, it would seem that even though the shadow test has been in common use in this country and France for a decade, the profession on the other side has not taken up the method to the extent that we have. In this country, I think that it may be said all of the progressive workers use it and do not consider the refracting of any case complete unless skiascopy has been called into service. Within a couple of years the acquisition of this method will be deemed as necessary by the foremost specialists of all the world as it is here. Six years ago I was astonished by the ignorance of the German oculists regarding skiascopy, as it was then, as now, foremost in my mind as being the principal objective method of measuring the eye. It is different now, and I understand that the shadow test is being generally taken up even in the clinics of Germany. Personally, I voice the opinion of its value given by your committee, and use it a number of times a day in my private practice.

In what cases should the shadow test be used? I should say in all patients for whom lenses are to be prescribed. I hold it to be an absolute necessity for the scientific refractionist to know in every instance the total refraction of the eye before putting on lenses. I would no more think of fitting spectacles to a presbyopic patient, by trying lenses at the near point alone without knowing his refraction, than I would of prescribing glasses in ametropia by one or more of the objective tests without recourse to the subjective method for confirmation.

The few instances in which skiascopy is unreliable or inapplicable are limited to those cases in which a definite visual zone can not be isolated. These eyes are the exception and yet where carefully used, in irregular corneal curvature, in conical cornea, in partial lenticular or corneal opacities and in very high grades of astigmatism, skiascopy is of value as a confirmatory test. "In nearly all eyes the result of careful skiascopic examination will give the best obtainable vision." 2

For skiascopic results to agree with those elicited by prolonged subjective tests, the examination should be conducted in the visual line, the refraction of the macula only being measured. Skiascopy measures the static refraction of the eye when applied in the visual zone, exactly as it is at the time of the examination, whether modified by the accommodation or not. Thus, in young persons, as a rule, a mydriatic should be used. Skiascopy is of value in proving of measurements found by the other methods, just like the proving of an example in vulgar fractions: By again applying the shadow test with the combination of lenses decided upon from the trial case. It may thus be used as a check upon the other methods and upon itself.

Aside from the difficulty of acquiring the technique, (although not nearly so much time is takt $n$ by the ty ro in learning it as is necessary for accurate ophthalmoscopic measurement), I would consider that the want of proper facilities for placing correcting lenses befure the patient has been one of the greatest obstacles to the general adoption of the method. If 
it be remembered that but one meridian can be considered at a time, and that the neutralization or reversal of the shadow movement by spherical lenses accomplishes the same purpose for the meridian under observation as cylindrical lenses, much of the difficulty is done away with. The placing and replacing of lenses in a trial frame is very consumptive of time, and the test case itself occupies too much space in the ophthalmoscopic dark room, which is usually but a closet.

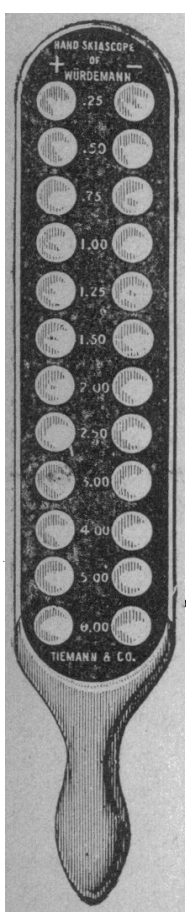

To obviate these disadvantages, Doyne, ${ }^{3}$ of Oxford, Eng., in the Ophthalmic Section of the International Congress held in Washington in 1887, exhibited a large rotary disk containing many plus and minus lenses. Burnett's and my own $n^{5}$ rotary disks, modifications of this, were more simple, but mine at least has been laid on the shelf for several years, as being too cumbersome. The simple hand skiascope, ${ }^{6}$ here shown has all their advantages and the model I present has been used in my work several times a day for nearly four years, and I understand has been given a place by a number of our colleagues. This you see is a simple blade of hard rubber which contains twelve plus and twelve minus lenses. The patient raises and lowers the instrument at the word of the examiner until the neutralizing lens is found, the strength of which is always plus $.75 \mathrm{D}$. greater than that of the actual ocular refraction in this meridian, and this over-correction should be allowed. The sphericals found by direct ophthalmoscopic examination are usually worn by the patient in a separate frame behind the instrument. I find it convenient to have a couple of pairs of reversible spectacle frames in my dark room containing the one $a+1$. D. and +2 . D. lens, and the other $\mathrm{a}+3 . \mathrm{D}$. and $\mathrm{a}+4$. D. lens; the strength of which when used for higher degrees of hyperopia is added to that of the instrument.

This simple instrument overcomes the principal difficulty in the use of the method. Thus skiascopic examination as practised by myself and colleagues takes but a minute or two, the test itself giving math. ematical data from which scientific conclusions are reached.

805 Grand Avenue.

REFERENCES.

1 Advt. Philadelphía Polyclinic. 1894

2 Report Special Committee, Section on Ophthalmology, Amer. Med Asso. Transactions. 1894

3 Doyne, Trans. Internat. Med. Cong. Washington, 1887.

4 Burnett, Swan M. Skiascopic Disk. Advt. Queen \& Co. 1890.

5 Würdemann, H. V., "The Use of Skiascopy." Amer. Jour. Ophth, May, 1890 .

6 Würdemann, H. V., "A Simple Skiascope." 'Amer. Jour. Ophth. Aug, 1891.

Sewage Disposal by Groeodiles. - One of the representatives of Ceylon at the Columbian Exposition is said to have proposed a plan for the "destruction of the offensive matter now passing into and festering in the river and imperiling the health and lives of the citizens, without necessitating any alteration of the existing system of sewerage." The plan of the Ceylonese "sanitarian" is to construct at the sewer outfalls, suitable enclosures in which to rear and keep "crocodiles and alligators to serve as scavengers-a service which, from the fact of their being omnivorous, I am convinced they are eminently well qualified to render" The veracious chronicle in which this precious plan was pub lished is said to have viewed the matter seriously.

\section{THE VISUAL ZONE OF THE DIOPTRIC MEDIA AND ITS STUDY BY SKIASCOPY.}

Read in the Section on Ophthalmology, at the Forty-fifth Annual Meeting of the American Medical Association, held at San Francisco, Jume 5-8, 1894.

BY EDWARD JACKSON, A.M., M.D.

PROFESSOR OF DISEASES OF THE EYE IN THE PHILADELPHIA POLYCLINIC SURGEON TO WILIS EYE HOSPITAI. PHILADELPHIA, PA.

One is liable to get the impression that, in eyes with perfect vision, only focused light reaches the retina, although the incorrectness of such an impression is readily demonstrated in various ways.

If we close the lids in any strong light, we can demonstrate that sufficient light reaches the retina through them to give quite accurate quantitative perception; and about the same amount of this light reaches the retina through the lids and the sclera when the eyes are open and in use for distinct vision. The ability to light up the interior of the eye by oblique illumination through the sclera or iris, we have all demonstrated. But skiascopy has shown that even through the pupil, always in the dilated pupil, often in the pupil as contracted by the ordinary daylight, a considerable part of the light entering, often the greater portion of it, reaches the retina unfocused.

While a flood of such unfocused light renders very accurate vision slightly more difficult and tiresome, it does not prevent it. The conditions may be likened to those that are obtained when a convex lens is held so as to form an image on a piece of paper in a well lighted room. A large part of the light reaching the paper is not focused light, and the image formed is on that account less vivid, yet more careful examination shows that it is comparatively distinct and quite complete. When the mixture of diffused and focused light falls on the retina, the former seems to interfere still less with the perception of the image formed by the latter, so that a comparatively small proportion of accurately focused light with a much larger amount of uniformly diffused light will still enable one to see distinctly.

The portions of the dioptric media through which the light is perfectly focused upon the retina I have termed the visual zone. Existence in the pupil of a visual and extra-visual zone, with some of the facts dependent thereon, have been impressed on me by prolonged use of the shadow-test, and I am conscious of having very slowly come to some realization of their full significance and importance. They have a most important influence upon the practical application of all methods for the measurement of ametropia, but the exact nature and significance of that. influence is only revealed by skiascopy.

The fact that we are able to see by the focused light in the presence of much that is unfocused, enables us to use the subjective method; and the fact that only the focused light impresses consciousness by recognizable sensations has, in a large proportion of cases removed the subjective test from the inaccuracies and uncertainties that would otherwise be due to the admission of light through the extra-visual zone.

The existence of an extra-visual zone presenting refractive conditions entirely different from those of the visual zone, is a great obstacle to accuracy with the direct method of ophthalmoscopy. With skia- 